International Journal of Modern Physics A

(C) World Scientific Publishing Company

\title{
Understanding open-charm mesons
}

\author{
J. Vijande ${ }^{1,2 *}$, F. Fernández ${ }^{1}$, A. Valcarce ${ }^{1}$. \\ ${ }^{1}$ Grupo de Física Nuclear and IUFFyM, Universidad de Salamanca, E-37008 Salamanca, Spain \\ ${ }^{2}$ Dpto. de Física Teórica and IFIC, Universidad de Valencia - CSIC, E-46100 Burjassot, \\ Valencia, Spain
}

\begin{abstract}
We present a theoretical framework that accounts for the new $D_{J}$ and $D_{s J}$ mesons measured in the open-charm sector. These resonances are properly described if considered as a mixture of conventional $P$-wave quark-antiquark states and four-quark components. The narrowest states are basically $P$-wave quark-antiquark mesons, while the dominantly four-quark states are shifted above the corresponding two-meson threshold. We study the electromagnetic decay widths as basic tools to scrutiny their nature.
\end{abstract}

During the last few years, heavy meson spectroscopy is living a continuous excitation due to the discovery of several new charmed mesons. Three years ago BABAR Collaboration reported the observation of a charm-strange state, the $D_{s J}^{*}(2317)^{1}$, that was later on confirmed by CLEO ${ }^{2}$ and Belle Collaborations 3 . Besides, BABAR had also pointed out to the existence of another charm-strange meson, the $D_{s J}(2460) \sqrt{1}$. This resonance was measured by CLEO ${ }^{2}$ and confirmed by Belle 3 . Belle results are consistent with the assignments of $J^{P}=0^{+}$for the $D_{s J}^{*}(2317)$ and $J^{P}=1^{+}$for the $D_{s J}(2460)$. However, although these states are well established, they present unexpected properties quite different from those predicted by quark potential models. If they would correspond to standard $P$-wave mesons made of a charm quark, $c$, and a strange antiquark, $\bar{s}$, their masses would be larger, around $2.48 \mathrm{GeV}$ for the $D_{s J}^{*}(2317)$ and $2.55 \mathrm{GeV}$ for the $D_{s J}(2460)$. They would be therefore above the $D K$ and $D^{*} K$ thresholds, respectively, being broad resonances. However the states observed by BABAR and CLEO are very narrow, $\Gamma<4.6 \mathrm{MeV}$ for the $D_{s J}^{*}(2317)$ and $\Gamma<5.5 \mathrm{MeV}$ for the $D_{s J}(2460)$.

The intriguing situation of the charm-strange mesons has been translated to the nonstrange sector with the Belle observation 4 of a nonstrange broad scalar resonance, $D_{0}^{*}$, with a mass of $2308 \pm 17 \pm 15 \pm 28 \mathrm{MeV} / \mathrm{c}^{2}$ and a width $\Gamma=$ $276 \pm 21 \pm 18 \pm 60 \mathrm{MeV}$. A state with similar properties has been suggested by FOCUS Collaboration at Fermilab ${ }^{5}$ during the measurement of masses and widths of excited charm mesons $D_{2}^{*}$. This state generates for the open-charm nonstrange mesons a very similar problem to the one arising in the strange sector with the $D_{s J}^{*}(2317)$. If the $D_{0}^{*}(2308)$ would correspond to a standard $P$-wave meson made of a charm

*E-mail address: javier.vijande@uv.es 
Table 1. $c \bar{s}$ and $c \bar{n}$ masses (QM), in MeV. Experimental data (Exp.) are taken from Ref $\frac{9}{}$, except for the state denoted by a dagger that has been taken from Ref. 4 .

\begin{tabular}{|c|ccc||ccc|}
\hline$n L \quad J^{P}$ & State & QM $(c \bar{s})$ & Exp. & State & QM $(c \bar{n})$ & Exp. \\
\hline $1 S 0^{-}$ & $D_{s}$ & 1981 & $1968.5 \pm 0.6$ & $D$ & 1883 & $1867.7 \pm 0.5$ \\
$1 S 1^{-}$ & $D_{s}^{*}$ & 2112 & $2112.4 \pm 0.7$ & $D^{*}$ & 2010 & $2008.9 \pm 0.5$ \\
$1 P 0^{+}$ & $D_{s J}^{*}(2317)$ & 2489 & $2317.4 \pm 0.9$ & $D_{0}^{*}(2308)$ & 2465 & $2308 \pm 17 \pm 15 \pm 28^{\dagger}$ \\
$1 P 1^{+}$ & $D_{s J}(2460)$ & 2578 & $2459.3 \pm 1.3$ & $D_{1}(2420)$ & 2450 & $2422.2 \pm 1.8$ \\
$1 P 1^{+}$ & $D_{s 1}(2536)$ & 2543 & $2535.3 \pm 0.6$ & $D_{1}^{0}(2430)$ & 2546 & $2427 \pm 26 \pm 25$ \\
$1 P 2^{+}$ & $D_{s 2}(2573)$ & 2582 & $2572.4 \pm 1.5$ & $D_{2}^{*}(2460)$ & 2496 & $2459 \pm 4$ \\
\hline
\end{tabular}

quark, $c$, and a light antiquark, $\bar{n}$, its mass would have to be larger, around $2.46 \mathrm{GeV}$. In this case, the quark potential models prediction and the measured resonance are both above the $D \pi$ threshold, the large width observed being expected although not its low mass.

The difficulties to identify the $D_{J}$ and $D_{s J}$ states with conventional $c \bar{n}$ mesons are rather similar to those appearing in the light-scalar meson sector $\underline{6}$ and may be indicating that other configurations are playing a role. $q \bar{q}$ states are more easily identified with physical hadrons when virtual quark loops are not important. This is the case of the pseudoscalar and vector mesons, mainly due to the $P$-wave nature of this hadronic dressing. On the contrary, in the scalar sector is the $q \bar{q}$ pair the one in a $P$-wave state, whereas quark loops may be in a $S$-wave. In this case the intermediate hadronic states that are created may play a crucial role in the composition of the resonance, in other words unquenching is important. This has been shown to be relevant for the proper description of the low-lying scalar mesons 7 .

In this work we have explored the same ideas for the understanding of the properties of the $D_{J}$ and $D_{s J}$ meson states. In non-relativistic quark models the wave function of a zero baryon number $(\mathrm{B}=0)$ hadron may be written as $|\mathrm{B}=0\rangle=\Omega_{1}|q \bar{q}\rangle+\Omega_{2}|q q \bar{q} \bar{q}\rangle+\ldots$. where $q$ stands for quark degrees of freedom and the coefficients $\Omega_{i}$ take into account the mixing of four- and two-quark states. The hamiltonian considering the mixing between both configurations could be described using the ${ }^{3} P_{0}$ model, however, since this model depends on the vertex parameter, we prefer in a first approximation to parametrize this coefficient by looking to the quark pair that is annihilated and not to the spectator quarks that will form the final $q \bar{q}$ state. Therefore we have taken $V_{q \bar{q} \leftrightarrow q q \bar{q} \bar{q}}=\gamma$. Further details about the formalism and the constituent quark model used are given in Refs. 78 .

A thoroughly study of the full meson spectra has been presented in Ref. $[$. The results for the open-charm mesons are resumed in Table 1 It can be seen how the open-charm states are easily identified with standard $c \bar{n}$ mesons except for the cases of the $D_{s J}^{*}(2317)$, the $D_{s J}(2460)$, and the $D_{0}^{*}(2308)$. This is a common behavior of almost all quark potential model calculations 10 . In a similar manner, quenched lattice NRQCD predicts for the $D_{s J}^{*}(2317)$ a mass of $2.44 \mathrm{GeV} 11$, while using relativistic charm quarks the mass obtained is $2.47 \mathrm{GeV}$ [12] Unquenched lattice QCD calculations of $c \bar{s}$ states do not find a window for the $D_{s J}^{*}(2317)$, supporting the difficulty of a $P$-wave $c \bar{s}$ interpretation. 
Table 2. Probabilities (P), in \%, of the wave function components and masses (QM), in MeV, of the open-charm mesons once the mixing between $q \bar{q}$ and $q q \bar{q} \bar{q}$ configurations is considered. Experimental data are taken from Ref ${ }^{9}$ except for the state denoted by a dagger that has been taken from Ref $\underline{4}$.

\begin{tabular}{|c|c||c|cc||c|c|}
\hline \multicolumn{5}{|c||}{$I=0$} & \multicolumn{2}{c||}{$I=1 / 2$} \\
\hline \multicolumn{2}{|c|}{$J^{P}=0^{+}$} & \multicolumn{3}{c||}{$J^{P}=1^{+}$} & \multicolumn{2}{|c|}{$J^{P}=0^{+}$} \\
\hline $\mathrm{QM}$ & 2339 & $\mathrm{QM}$ & 2421 & 2555 & $\mathrm{QM}$ & 2241 \\
Exp. & $2317.4 \pm 0.9$ & Exp. & $2459.3 \pm 1.3$ & $2535.3 \pm 0.6$ & Exp. & $2308 \pm 17 \pm 15 \pm 28^{\dagger}$ \\
\hline $\mathrm{P}(c n \bar{s} \bar{n})$ & 28 & $\mathrm{P}(c n \bar{s} \bar{n})$ & 25 & $\sim 1$ & $\mathrm{P}(c n \bar{n} \bar{n})$ & 46 \\
$\mathrm{P}\left(c \bar{s}_{13}\right)$ & 71 & $\mathrm{P}\left(c \bar{s}_{11}{ }^{1}\right)$ & 74 & $\sim 1$ & $\mathrm{P}\left(c \bar{n}_{1 P}\right)$ & 53 \\
$\mathrm{P}\left(c \bar{s}_{2^{3} P}\right)$ & $\sim 1$ & $\mathrm{P}\left(c \bar{s}_{13}\right)$ & $\sim 1$ & 98 & $\mathrm{P}\left(c \bar{n}_{2 P}\right)$ & $\sim 1$ \\
\hline
\end{tabular}

Using for the $q q$ interaction the parametrization of Ref! ${ }^{[}$, the results obtained for the $c n \bar{s} \bar{n}$ configuration are 2731 and $2699 \mathrm{MeV}$ for the $J^{P}=0^{+}$with $I=0$ and $I=1$, and 2841 and $2793 \mathrm{MeV}$ for the $J^{P}=1^{+}$with $I=0$ and $I=1$. For the $c n \bar{n} \bar{n}$ configuration with $I=1 / 2$ the energy is $2505 \mathrm{MeV}$. The $I=1$ and $I=0$ states are far above the corresponding strong decaying thresholds and therefore should be broad, what rules out a pure four-quark interpretation of the new open-charm mesons.

As outlined above, for $P$-wave mesons the hadronic dressing is in a $S$-wave, thus physical states may correspond to a mixing of two- and four-body configurations. In the isoscalar sector, the $c n \bar{s} \bar{n}$ and $c \bar{s}$ states get mixed, as it happens with $c n \bar{n} \bar{n}$ and $c \bar{n}$ for the $I=1 / 2$ case. The parameter $\gamma$ has been fixed to reproduce the mass of the $D_{s J}^{*}(2317)$ meson, $\gamma=240 \mathrm{MeV}$. The results obtained are shown in Table 2. Let us first analyze the nonstrange sector. The ${ }^{3} P_{0} c \bar{n}$ pair and the $c n \bar{n} \bar{n}$ have a mass of $2465 \mathrm{MeV}$ and $2505 \mathrm{MeV}$, respectively. Once the mixing is considered one obtains a state at $2241 \mathrm{MeV}$ with $46 \%$ of four-quark component and $53 \%$ of $c \bar{n}$ pair. The lowest state, representing the $D_{0}^{*}(2308)$, is above the isospin preserving threshold $D \pi$, being broad as observed experimentally. The mixed configuration compares much better with the experimental data than the pure $c \bar{n}$ state. The orthogonal state appears higher in energy, at $2713 \mathrm{MeV}$, with and important four-quark component.

Concerning the strange sector, the $D_{s J}^{*}(2317)$ and the $D_{s J}(2460)$ are dominantly $c \bar{s} J=0^{+}$and $J=1^{+}$states, respectively, with almost $30 \%$ of four-quark component. Such component is responsible for the shift of the mass of the unmixed states to the experimental values below the $D K$ and $D^{*} K$ thresholds. Being both states below their isospin-preserving two-meson threshold, the only allowed strong decays to $D_{s}^{*} \pi$ would violate isospin and are expected to have small widths $O(10) \mathrm{keV} 1314$. As a consequence, they should be narrower than the $D_{s 2}(2573)$ and $D_{s 1}(2536)$, opposite to what it is expected from heavy quark symmetry. The second isoscalar $J^{P}=1^{+}$state, with an energy of $2555 \mathrm{MeV}$ and $98 \%$ of $c \bar{s}$ component, corresponds to the $D_{s 1}(2536)$. Regarding the $D_{s J}^{*}(2317)$, it has been argued that a possible $D K$ molecule would be preferred with respect to an $I=0 \mathrm{cn} \bar{s} \bar{n}$ tetraquark, what would anticipate an $I=1 c n \bar{s} \bar{n}$ partner nearby in mass 15 . Our results confirm the last argument, the vicinity of the isoscalar and isovector tetraquarks, however, the re- 
Table 3. Electromagnetic decay widths, in $\mathrm{keV}$, for the $D_{s J}^{*}(2317)$ and $D_{s J}(2460)$ (QM), compared to the results of two different quark models based only on $q \bar{q}$ states. To compare with the experimental data by CLEO and Belle we have assumed for $\Gamma\left(D_{s}^{*+} \pi^{0}\right) \approx \Gamma\left(D_{s}^{+} \pi^{0}\right) \approx 10 \mathrm{keV}$ as estimated in Ref. 14.

\begin{tabular}{|c|ccc|cc|}
\hline & \multicolumn{3}{|c|}{ Quark models } & \multicolumn{2}{c|}{ Experiments } \\
Transition & QM & Ref $[3]$ & Ref 14 & CLEd & Belle \\
\hline$D_{s J}^{*}(2317) \rightarrow D_{s}^{*+} \gamma$ & 1.6 & 1.74 & 1.9 & $<0.59$ & $<1.8$ \\
$D_{s J}(2460) \rightarrow D_{s}^{*+} \gamma$ & 0.06 & 4.66 & 5.5 & $<1.6$ & $<3.1$ \\
$D_{s J}(2460) \rightarrow D_{s}^{+} \gamma$ & 6.7 & 5.08 & 6.2 & $<4.9$ & $5.5 \pm 1.3 \pm 0.8$ \\
\hline
\end{tabular}

stricted coupling to the $c \bar{s}$ system allowed only for the $I=0$ four-quark states opens the possibility of a mixed nature for the $D_{s J}^{*}(2317)$ while the $I=1 \mathrm{~J}=0^{+}$and $J=1^{+}$four-quark states appear above $2700 \mathrm{MeV}$ and cannot be shifted to lower energies.

Apart from the masses, the structure of the $D_{s J}^{*}(2317)$ and the $D_{s J}(2460)$ mesons could be scrutinied also through the study of their electromagnetic decay widths. We compare in Table 3 our results with different theoretical approaches and the experimental limits reported by Belle and CLEO. The main difference is noticed in the suppression predicted for the $D_{s J}(2460) \rightarrow D_{s}^{*+} \gamma$ decay as compared to the $D_{s J}(2460) \rightarrow D_{s}^{+} \gamma$. A ratio $D_{s J}(2460) \rightarrow D_{s}^{+} \gamma / D_{s J}(2460) \rightarrow D_{s}^{*+} \gamma \approx$ $1-2$ has been obtained assuming a $q \bar{q}$ structure for both states 1314 (what seems incompatible with their properties). We find a larger value, $D_{s J}(2460) \rightarrow D_{s}^{+} \gamma / D_{s J}(2460) \rightarrow D_{s}^{*+} \gamma \approx 100$, due to the small $1^{3} P_{1} c \bar{s}$ probability of the $D_{s J}(2460)$. A similar enhancement has been obtained in Ref. $\frac{16]}{}$ in the framework of light-cone QCD sum rules.

This work has been partially funded by Ministerio de Ciencia y Tecnología under Contract No. FPA2004-05616, by Junta de Castilla y León under Contract No. SA104/04, and by Generalitat Valenciana under Contract No. GV05/276.

\section{References}

1. B. Aubert et al., [BABAR Collaboration], Phys. Rev. Lett. 90, 242001 (2003).

2. D. Besson et al., [CLEO Collaboration], Phys. Rev. D 68, 032002 (2003).

3. Y. Mikani et al., [Belle Collaboration], Phys. Rev. Lett. 92, 012002 (2004).

4. K. Abe et al., [Belle Collaboration], Phys. Rev. D 69, 112002 (2004).

5. J.M. Link et al., [FOCUS Collaboration], Phys. Lett. B 586, 11 (2004).

6. G.S. Bali, Phys. Rev. D 68, 071501(R) (2003).

7. J. Vijande et al., Phys. Rev. D 72, 034025 (2005).

8. J. Vijande, F. Fernández, and A. Valcarce, J. Phys. G 19, 2013 (2005).

9. S. Eidelman et al., Phys. Lett. B 592, 1 (2004).

10. S. Godfrey and R. Kokoski, Phys. Rev. D 43, 1679 (1991).

11. J. Hein et al., Phys. Rev. D 62, 074503 (2000).

12. P. Boyle, [UKQCD Collaboration], Nucl. Phys. B (Proc. Supp.) 63, 314 (1998).

13. W.A. Bardeen, E.J. Eichten, and C.T. Hill, Phys. Rev. D 68, 054024 (2003).

14. S. Godfrey, Phys. Lett. B 568, 254 (2003).

15. T. Barnes, F.E. Close, and H.J. Lipkin, Phys. Rev. D 68, 054006 (2003).

16. P. Colangelo, F. de Fazio, and A. Ozpineci, Phys. Rev. D 72, 074004 (2005). 